\title{
Retrospective analysis of prognostic value of neutrophils to lymphocyte ratio and platelet count in patients with colorectal carcinoma
}

\author{
Mohamed Elbassiouny, Dina Ragab, Ghada Refaat, Suhad A. Ali* \\ Department of clinical oncology and nuclear medicine, faculty of medicine of Ain Shams University, Cairo, Egypt
}

Received: December 2, 2019

DOI: $10.5430 /$ jst.v10n1p16
Accepted: December 20, 2019 Online Published: February 10, 2020

URL: https://doi.org/10.5430/jst.v10n1p16

\begin{abstract}
Background: Colorectal cancer (CRC) is the third most common cancer in men and second in women with 1.8 million new cases (1,026,000 men and 823, 3 women) and almost 881.000 deaths. Rates are substantially higher in males than in females Worldwide in 2018.

Aim of the work: In this retrospective study we aimed to evaluate the prognostic impact of baseline NLR and platelet count on the clinicopathological factors and outcome in patients of all stages Colorectal cancer treated from 1st of January 2014 to the end of December 2016 in Department of Clinical Oncology and Nuclear Medicine, Ain Shams University hospitals, Cairo, Egypt. Patients and methods: Out of 409 patient's medical records in the GI oncology unit, Ain Shams Clinical Oncology Department were reviewed from the period between 1st of January 2014 to 30 December 2016. Total neutrophils, lymphocytic, and platelets' counts were available for only 169 patients. Study ended in 1st of August 2018 with median period of follow up of 27.5 month, ranging between $1 / 1 / 2014$ to $1 / 8 / 2018$. All patients (169) were pathologically proven colorectal adenocarcinoma, with age ranging from 18-75 years old (median age: $55.5 \mathrm{yrs}$.)

Results: Out of 169 patients enrolled in this study, 124 patients were resectable and underwent curative surgeries, 44 patients tumour was right located and 80 patient's tumour located in the left sided colon. 45 patients were metastatic from the start Postoperative Platelets $\geq 310$ in our study was statistically significant regarding OS, PFS and DFS $(P$ values $<.001,<.001$ and $0.007)$ respectively. Pre-treatment platelet revealed more frequent thrombocytosis in metastatic group than locally advanced group, yet statistically was not significant $(P$ Value $=.066)$. Postoperative NLR $\geq 2$ was significant regarding OS, PFS and DFS among 169 enrolled patients $(P$ values $<.001, .002$ and <.001) respectively. In the multivariate analysis, elevated postoperative NLR was proven as both independent prognostic and predictor factor for DFS, PFS and OAS. (sig. $=.03, .03, \leq 0.001$ respectively). And platelet count is both independent prognostic factor and predictor for both PFS, OS with significance $=.04,=.03$ respectively).

Conclusion: Abnormal NLR ratio $(\geq 2)$ acting as a prognostic and predictor of decrease in DFS, PFS and OS in all patients groups. It also showed that abnormal platelet count $(\geq 310)$ is prognostic and predictor of significant decrease in PFS and OS. Multidisciplinary management is needed to aware surgeons about importance of adequate lymph node dissection, our study showed a statistically significant decrease in OAS in patients underwent inadequate LNs dissection.
\end{abstract}

Key Words: Neutrophils, Lymphocyte, Platelet count, Colorectal carcinoma

*Correspondence: Suhad A. Ali; Email: suhadmaidan89@ gmail.com; Address: Department of clinical oncology and nuclear medicine, faculty of medicine of Ain Shams University, Cairo, Egypt. 